\title{
Perspective
}

PERSPECTIVE Actualité en histoire de l'art

4 | 2006

La monographie d'artiste

\section{Un voyage dans la lumière : Rainer Werner Fassbinder}

\section{Marianne Dautrey}

\section{(2) OpenEdition}

1 Journals

Édition électronique

URL : http://journals.openedition.org/perspective/4232

DOI : $10.4000 /$ perspective.4232

ISSN : 2269-7721

Éditeur

Institut national d'histoire de l'art

\section{Édition imprimée}

Date de publication : 31 décembre 2006

Pagination : 649-652

ISSN : 1777-7852

\section{Référence électronique}

Marianne Dautrey, "Un voyage dans la lumière : Rainer Werner Fassbinder », Perspective [En ligne], 4| 2006, mis en ligne le 31 mars 2018, consulté le 01 octobre 2020. URL : http://

journals.openedition.org/perspective/4232 ; DOI : https://doi.org/10.4000/perspective.4232 
La monographie doit aussi parfois accepter de perdre son auteur, pour ne pas le perdre de vue.

1. Roberto Rossellini, (Adriano Aprà éd.), La télévision comme utopie, Paris, 2001.

2. Citons, dans l'actualité, la stupéfiante intégrale Norman McLaren éditée en 2006 par l'Office national du film du Canada (une exception historique puisque l'Office fut presque l'unique producteur de McLaren, ainsi restaurations et sorties d'inédits, de chutes et de tests filmés complètent-ils une intégrale véritable), et le grand nombre de films de Fassbinder dans un coffret de 18 DVD, rassemblés par la société française de distribution Carlotta Films.

3. Une chercheuse, qui a scruté plan par plan et ligne après ligne (entamant au passage sérieusement le mythe des films sans scénario, entretenu par l'auteur) l'ensemble des six films réalisés avec Ingrid Bergman (1951-1954), considérés comme un "polyptyque " qui fait sens, historiquement audelà des deux personnes en jeu (un rapport entre le nouvel actorat et la modernité), recense même patiemment, par exemple, entre autres documents utiles, les différentes éditions de cassettes vidéo qui témoignent des copies films qui ont pu circuler, avec leurs coupes ou leurs ajouts. Voir la plus philologique des monographies rosselliniennes : Elena Dagrada, Le variente trasparenti. I film con Ingrid Bergman di Roberto Rossellini, Milan, 2005, 478 p., illustrations noir et blanc, et six films découpés systématiquement pour le texte et l'image avec tableaux comparatifs de toutes les versions existantes.

4. Alain Bergala, "Les quatre vies de cinéma de Rossellini ", programme de la Cinémathèque française, janvier-février 2006, p. 28-31.

5. Ibidem.

Hervé Joubert-Laurencin, université Paris 7 - Denis Diderot, hjl@noos.fr

\section{Un voyage dans la lumière : Rainer Werner Fassbinder}

\section{Marianne Dautrey}

En 2005, le Centre George-Pompidou organisait une rétrospective complète de l'œuvre cinématographique de Rainer Werner Fassbinder. À cette occasion, a été traduite la monographie que Thomas Elsaesser avait consacrée au cinéaste, Fassbinder, un cinéaste d'Allemagne (Paris, 2005), tandis qu'en 2005-2006 les éditions Carlotta publiaient une série de quatre coffrets DVD qui, désormais, met à disposition 25 films de Fassbinder, accompagnés de courts-métrages de l'auteur, de documentaires sur ses films et d'extraits d'interviews (fig. 1). La monographie de Thomas Elsaesser marque la tentative d'embrasser l'ensemble des films de Fassbinder à l'intérieur d'un discours spéculatif et cohérent. Les coffrets, eux, présentent sinon l'intégralité de l'œuvre du cinéaste, du moins une sélection de films suffisamment variée pour esquisser une vision d'ensemble de celle-ci. Ne pouvant être autre chose qu'une juxtaposition de films et ne pouvant produire un discours immédiatement cohérent, ils affrontent à l'évidence un problème épineux : celui de faire percevoir les lignes de force qui traversent ces films et la trame qui se tisse de l'un à l'autre au travers d'une simple présentation de ces derniers. Les enjeux de chacune de ces deux présentations de la filmographie de Fassbinder se rejoignent donc : il s'agit de faire apparaître cette dernière comme une ouvre dans son sens plein. Et, dans cette perspective, elles se complètent aussi, se confirmant ou se corrigeant, et permettent un regard nouveau sur le travail du cinéaste.

Le travail du cinéaste Fassbinder fait œuvre d'une manière radicale et volontaire. Cela n'avait pourtant rien eu d'évident jusqu'ici. D'abord parce que ses films traversent des genres parfaitement hétérogènes, tantôt films noirs, tantôt mélodrames, tantôt films sur

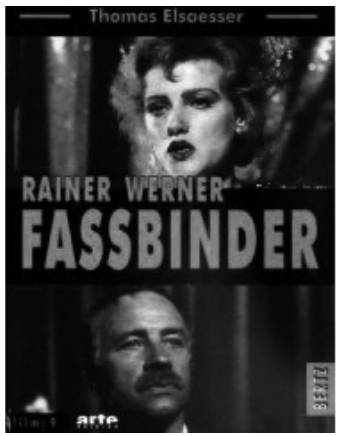

1. Couverture du coffret DVD Fassbinder. 
l'Allemagne, ensuite parce qu'ils ont été créés dans des économies de production radicalement différentes - au cours de sa carrière, le cinéaste, qui enchaînait film sur film, sans répit, est passé tour à tour du film confidentiel et expérimental à tout petit budget, tourné à la va-vite dans une économie pour ainsi dire autarcique avec sa troupe, au projet subventionné ou encore à la grosse production internationale grand public où étaient convoquées des stars hollywoodiennes. Et c'est encore en raison de la production prolifique du cinéaste qu'on a parfois perçu ses films comme des œuvres de circonstance, des confrontations polémiques qui réagissaient à chaque fois dans l'urgence à une situation donnée, qu'elle relève de la réalité politique du moment, d'une crise dans la vie du cinéaste ou d'une commande.

Et si les films de Fassbinder n'avaient pourtant jamais fait que répondre à un seul et même problème qui, lancinant et obsédant, revenait sans cesse, faisant de leur succession une variation continue, une fugue autour d'un seul thème, comme autant de réponses possibles à ce même problème ? Telle est l'hypothèse qu'avance et met à l'épreuve Thomas Elsaesser. Allant à l'encontre de la plupart de ses prédécesseurs, il renonce délibérément à prendre en compte la biographie de l'auteur et aborde ses films comme des entités qui tirent leur sens non pas directement des circonstances de leur création mais d'une démarche d'objectivation, d'une réflexion spéculative qui, de film en film, se fraie un chemin, sans doute accidenté mais cohérent, et qui trace une ligne au point de sembler raconter une histoire continue tout au long de son développement.

Si Thomas Elsaesser souligne une continuité dans l'œuvre du cinéaste, il ouvre néanmoins une perspective en faisant des films de Fassbinder le lieu d'une élaboration spéculative, politique et existentielle et, à ce titre, s'inscrit également en faux à l'égard de tous ceux qui, certes, en avaient perçu la cohérence mais avaient voulu y voir un tout autonome. À l'instar, par exemple, de ceux qui identifiaient, dans cette succession de narrations, un équivalent cinématographique et allemand d'une Comédie humaine, interprétant la suite des films comme autant de tableaux réalistes et achevés des facettes politiques, sociales, culturelles et géographiques du pays. Comme pour les conforter dans cette interprétation, Fassbinder lui-même affirmait : " Je ferai des films jusqu'à ce que j'aie conduit mon histoire de la RFA jusqu'ici et aujourd'hui ". Ce qui fondait certains d'entre eux à ériger en outre ce cinéaste qui, à leurs yeux, entendait embrasser toute l'histoire de son pays, en représentant, certes critique, mais non moins représentant de l'Allemagne de l'Ouest au même titre qu'un Balzac avait endossé ce rôle dans la France du XIX siècle. Mais, comme le souligne Thomas Elsaesser, pour Fassbinder, il ne s'agissait pas tant d'écrire une histoire de son pays que de la " conduire jusqu'ici et aujourd'hui ", c'est-à-dire de l'éprouver à partir de ses enjeux actuels ou encore d'en poursuivre les ramifications jusque dans ses manifestations présentes. Or, dans cette perspective, ce n'est pas une histoire de l'Allemagne de l'Ouest, pas plus qu'une description réaliste du pays qu'a élaborée Fassbinder, mais une archéologie de son présent. La distinction n'est pas anodine, car elle implique une redéfinition tout autant du statut de l'œuvre que de celui de son auteur.

Thomas Elsaesser identifie un dispositif d'énonciation qui met en jeu le cinéaste, la caméra et le spectateur et reconduit le film, dans le même temps, au présent de son tournage et du visionnage du spectateur. Ce qui exclut de considérer ses films comme des touts autonomes, mais en fait bien plutôt des actions directes, des interventions du cinéaste auprès de ses spectateurs et donc dans le présent de son pays. Ce sont, dans les films du début de la carrière du cinéaste (Le bouc, 1969 ; Le soldat américain, 1970 ; Les pionniers d'Ingoldstadt, 1971 ; Effi Briest, 1972), des images frontales privées de profondeur de champ, prises dans une narration syncopée qui isole chaque scène comme un tableau en suspens, provisoire et incomplet, où les personnages font face au spectateur sans que ce dernier puisse identifier de manière univoque la raison et la fin de leur présence ni même l'objet qu'embrasse leur regard. De cette manière, dès ses premiers films, Fassbinder ouvre une brèche qui les renvoie à la fois au présent de leur élaboration et à celui du visionnage du spectateur et, par ce procédé, il fait de ses personnages non pas des protagonistes à part entière mais de simples apparitions dans la lumière des projecteurs ou dans le champ de vision de la caméra, autrement dit : de purs phénomènes - cinématographiques.

Ses apparitions, qui viennent se plaquer sur une surface sans profondeur, au même titre qu'elles sont privées d'une histoire au cours de laquelle elles auraient pu se construire une identité fixe, tra- 
versent une crise identitaire dans les films de sa seconde période, le plus souvent des mélodrames. Soit qu'elles ignorent ou refoulent à la fois leur identité et celle de ceux qui les entourent ( $L a$ peur de la peur, 1975 ; Martha, 1974), soit que, prenant conscience de ce refoulement, elles tentent de s'en construire une (Maman Küster s'en va au ciel, 1975) ou encore que leur position, leur identité sociale, donc leur image, se modifient au cours du film (Tous les autres s'appellent Ali, 1974 ; Le marchand de quatre saisons, 1972 ; Le droit du plus fort, 1975), elles se trouvent au cour de tensions antagoniques, que Thomas Elsaesser nomme "double bind " et interprète comme un champ de regards, lesquels agissent comme autant d'injonctions identitaires contradictoires. Dans ces films, où le récit retrouve une certaine continuité, une identification du spectateur devient possible. Partiellement du moins car, très vite, celle-ci se heurte au décentrement du personnage qui, dans son fantasme d'identité, n'a de cesse de se modeler, de se transformer, de se défaire à travers les gestes de l'amour, du sacrifice, de l'exploitation et de la tyrannie et qui meurt de n'être pas vu ou d'être mal vu ou encore de vouloir être l'image de l'être aimé. Un trouble s'instaure qui fait vaciller le rapport entre voir et se montrer. Ce n'est pas tant le spectateur qui voit le personnage que ce dernier qui se montre, s'exhibe à lui. Et Fassbinder a beau cacher sa caméra dans l'embrasure d'une porte ou d'une fenêtre, jamais il ne place le spectateur en position de voyeur, puisque ce que celui-ci découvre alors est un être déjà en train de guetter un regard. Par ce processus de " mise en abyme", les personnages sont de nouveau relégués au statut d'images qui, pour exister, requièrent de manière explicite le regard d'un autre mais, dans le même temps, deviennent sujets de cette requête. De sorte que, dans les films de la dernière période, dans la trilogie allemande, (Le mariage de Maria Braun, Lola une femme allemande, Veronika Voss), dans Despair, L'année des treize lunes ou dans Berlin Alexanderplatz, ce sont les personnages eux-mêmes qui inventent une nouvelle économie identitaire, échangeant leur propre identité, se livrant à des dédoublements (Despair), à des altérations de leur corps et, par ce biais, de leur identité sexuelle. Ces films déploient alors un espace où les rôles font l'objet d'insolites contrats qui mettent en jeu tout autant des trafics de drogues, une commercialisation des corps qu'un don de soi (Le mariage de Maria Braun, L'année des treize lunes, Lili Marlène), et cela, jusqu'au sacrifice de soi, jusqu'au point où les personnages consument leur existence et réalisent avec stupeur qu'il ne subsiste d'eux-mêmes plus que des décombres épars.

Et il y a, de film en film, comme un approfondissement vertigineux du décentrement des personnages. $\mathrm{Si}$, dans la première période, le dispositif triangulaire qui se met en place entre le cinéaste, la caméra et le spectateur avait pour fonction essentielle de produire un effet de distanciation, sur le modèle brechtien, il contribue, par la suite, à prendre le spectateur à témoin dans cet approfondissement de la spirale centrifuge qui entraîne le personnage à se perdre pour, en toute fin, le faire entrer dans une économie de l'excès, de la perte et du sacrifice de soi ou, à l'inverse, de l'exploitation et de la destruction, et devenir un objet médiatisé étranger à soi-même, comme Lili Marlène ou Erwin/Elvira dans L'année des treize lunes ou Maria Braun.

Derrière ce vertigineux mouvement d'aliénation, derrière cette trame, il y aurait, nous dit Thomas Elsaesser, la volonté de Fassbinder de mettre à l'épreuve d'une Allemagne d'abord d'après-guerre, puis des trente glorieuses et, enfin, des années de plomb le scénario hollywoodien de la constitution œdipienne du sujet, à partir du nœud généalogique. Comme si Fassbinder, inlassablement, avait répété, en l'approfondissant, cette expérience qui consiste à exposer le sujet allemand et l'histoire allemande sous les projecteurs du "cinéma", comme si, à chaque fois, il fallait faire ce constat que, à l'instar du mythe qui ne peut plus se constituer en récit autonome et fondateur, le scénario ne peut plus que se fissurer pour s'ouvrir à un espace situé non pas seulement hors des formes nobles du cinéma (la série télévisée, par exemple) mais aussi hors de la fiction, et renvoyer au geste de son élaboration. Ce sont ces personnages de la première période qui, relégués au statut de simples phénomènes, créatures purement cinématographiques, s'épuisent à emprunter des postures d'autres personnages de cinéma, comme ce soldat américain qui ne semble sortir d'un film de Lang ou de Raoul Walsh que pour mieux trahir l'imposture dont il n'est que le vecteur, et se découvre sans autre raison d'être. Sans cesse les films de Fassbinder réfèrent au cinéma, ils citent d'autres films, mais, pour finir, c'est encore les siens qu'ils citent le plus fréquemment comme si, progressivement, il réussissait à construire un espace cinématographique cohérent autre que l'espace hollywoodien, un espace intermédiaire entre le film télévisé et le film 


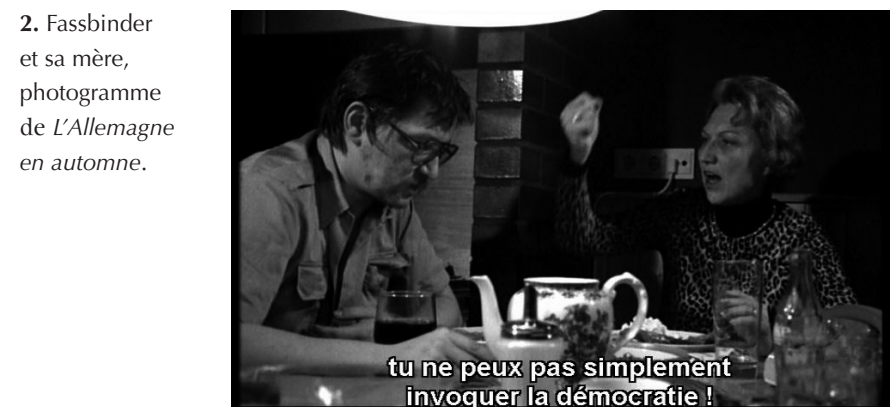

d'auteur, où le personnage n'aurait plus de généalogie, plus de père et mère, inventerait d'autres modes utopiques d'existences possibles et déploierait un autre espace politique, qui s'incarne dans ses fictions mais aussi dans la relation inédite qu'il instaure entre cinéaste, caméra et spectateur.

Les apparitions de Fassbinder au sein de ses films sont tout autant le signe que la mise en pratique de cette politique (fig. 2). Et il apparaît à l'écran, en effet, soit en tant que personnage fictionnel comme dans Le droit du plus fort, soit dans un entre-deux, en tant que passeur (Le mariage de Maria Braun), soit en tant que Fassbinder lui-même dans L'Allemagne en automne. Là, il ne représente rien d'autre que luimême qui, d'une part, met sa parole à l'épreuve de celle de sa mère (celle-ci incarnant le citoyen allemand quelconque), et d'autre part exhibe son propre corps, un corps qui se dénude et s'effondre peu à peu, traversé par les spasmes de l'angoisse, de la peur face à un État qui, par sa violence, génère une violence diffuse, relayée par ses sujets, qu'il s'agisse de terroristes, étrangement suicidés dans leur cellule, ou de citoyens anonymes qui, à l'instar de sa mère, renoncent à leur propre parole et réclament la présence d'un "Führer", certes " doux, raisonnable et juste ", mais non moins autoritaire, figure $d^{\prime}$ un père entre les mains duquel ils seraient prêts à remettre leur propre personne...

Thomas Elsaesser embrasse ainsi toute l'œuvre de Fassbinder dans sa cohérence, mais cependant il la fissure, pour la présenter comme une œuvre ouverte, expérimentale qui aurait été vouée à se prolonger encore si elle n'avait été interrompue par une mort précoce. C'est sur ce point précisément que la confrontation avec l'édition des films réalisée par Carlotta apparaît intéressante. Carlotta rapproche, en les juxtaposant, des films de Fassbinder lui-même, issus de différentes époques de sa production. Par ce procédé, il institue le cinéma de Fassbinder comme son propre commentateur, comme son propre critique. Par exemple, en plaçant en regard Martha et La peur de la peur, ou encore La troisième génération et Le petit chaos, il ne révèle pas seulement une continuité de l'œuvre, des premiers films jusqu'aux derniers, il montre aussi que ceux-ci décrivent une variation continue, comme si, dans sa démarche spéculative, Fassbinder n'avait eu de cesse de combiner et de recombiner ses données entre elles et, de cette manière, de montrer leur vacillement, leur ambivalence. Si cette présentation rejoint et confirme le discours de Thomas Elsaesser, elle n'en casse pas moins la systématicité de ce dernier qui occulte le fait que si, en toute fin, les films de Fassbinder renvoient au geste de leur créateur, il n'y a pas forcément une continuité entre l'intention de ce créateur et ses réalisations, et que cette ambivalence qui frappe les personnages et les fait devenir des données de combinaisons possibles touche tout autant leur créateur qui le montre en s'instituant lui-même comme l'un de ses propres personnages. Sans doute est-ce aussi cette systématicité qui l'empêche de voir que dans L'année des treize lunes, Erwin/Elvira n'est pas un enfant du Lebensborn, comme il l'affirme, par un souci de symétrie face au personnage du juif incarné par Anton Saitz, mais un enfant illégitime : à l'encontre des juifs, l'Allemagne nazie n'a pas tant engendré des Aryens que des enfants illégitimes privés de passé, d'histoire, de généalogie et devenus impossible d'adopter, à l'instar des personnages de Fassbinder. Ceux-ci ont beau alors s'inventer d'autres formes d'existence, ils n'en périssent pas moins, privés de fondement, se dissolvant sous l'effet d'une surexposition à la lumière des projecteurs comme Veronika Voss, se désagrégeant comme Maria Braun, se désintégrant comme Erwin/Elvira ou encore Franz Biberkopf... Mais sans doute la présence de la propre mère de Fassbinder dans presque tous ses films sur le même plan que tous les autres personnages et au même titre que Fassbinder lui-même est-elle le symptôme le plus éloquent de cette mise à plat du nœud identificatoire œdipien. Cependant, devenue personnage, si elle ne peut plus fonder aucun récit généalogique, elle témoigne de la possibilité d'un autre récit - quant aux autres personnages, ils meurent d'avoir tenté d'éprouver ce récit.

NB. Le titre de cet article fait écho à la traduction en français du titre de la version allemande de Despair.

Marianne Dautrey, Université Paris-I, marianne.dautrey@noos.fr 